PROCEEDINGS OF THE

AMERICAN MATHEMATICAL SOCIETY

Volume 132, Number 7, Pages 1963-1971

S 0002-9939(04)07159-X

Article electronically published on February 6, 2004

\title{
LOCAL EXISTENCE OF $\mathcal{K}$-SETS, PROJECTIVE TENSOR PRODUCTS, AND ARENS REGULARITY FOR $A\left(E_{1}+\cdots+E_{n}\right)$
}

\author{
COLIN C. GRAHAM
}

(Communicated by Andreas Seeger)

\begin{abstract}
Theorem. If $X_{1}, \ldots, X_{n}$ are perfect compact subsets of the locally compact metrizable abelian group, then there are pairwise disjoint perfect subsets $Y_{1} \subseteq X_{1}, \ldots, Y_{n} \subseteq X_{n}$ such that (i) $Y_{j}$ is either a Kronecker set or (ii) for some $p_{j} \geq 2, Y_{j}$ is a translate of a $K_{p_{j}}$-set all of whose elements have order $p_{j}$, and (iii) $A\left(Y_{1}+\cdots+Y_{n}\right)$ is isomorphic to the projective tensor product $C\left(Y_{1}\right) \hat{\otimes} \cdots \hat{\otimes} C\left(Y_{n}\right)$.

This extends what was previously known for groups such as $\mathbb{T}$ or for the case $n=2$ to the general locally compact abelian group. Old results concerning the local existence of Kronecker and $K_{p}$-sets are improved.
\end{abstract}

\section{INTRODUCTION}

This paper brings together ideas of Varopoulos [13] and Kahane [7] for the statement and proof of the Theorem stated in the Abstract. (Definitions are given later in this section.)

Parts (i)-(ii) of that Theorem are given for the circle group [7, p. 92]; the entire Theorem is given for the case $n=2$ and the general metrizable locally compact abelian group in 13, Theorem 4.3.3]. Varopoulos also gives the equivalence of $A\left(E_{1}+\cdots+E_{n}\right) \cong C\left(E_{1}\right) \hat{\otimes} \cdots \hat{\otimes} C\left(E_{n}\right)$ for general $n$ in [13, 4.2.1-4.2.2]. However, [13, Thm 4.3.3] may have been overlooked (compare [6, 41.5] and [10, p. 213]), and part of the purpose of this note is to call attention to the most general form of the result, as well as to give a somewhat detailed proof of a slightly stronger result than [13. We adapt the quasi-all argument in [7, pp. 91-92], due originally to Kaufman [8].

The Theorem has the following corollary, which improves the standard [6, 41.5] by replacing "open $X$ " with "non-void perfect $X$ ".

Corollary 1.1. Let $G$ be a metrizable locally compact abelian group. Every nonvoid perfect $X \subset G$ contains a Cantor subset that is either a Kronecker set or a translate of a $K_{p}$-set all of whose elements have order $p$.

Received by the editors September 12, 2002 and, in revised form, December 23, 2002.

2000 Mathematics Subject Classification. Primary 43A15, 43A10; Secondary 46L10.

Key words and phrases. Arens regularity, bidual, Kronecker set, $K_{p}$-set, locally compact abelian groups, projective tensor product, quotients of the Fourier algebra, set sums, tensor algebra.

Preprints of a draft of this paper were circulated under the title "Arens regularity and related matters for $A(E+F)$ ". 
Organization of this paper. Some further corollaries are given in this section. Each corollary is followed by comments pertinent to its statement. Then definitions used later are given in the remainder of this section, followed by the statement of Proposition 1.6, which (when proved) suffices for the proof of parts (i)-(ii) of the Theorem. Part (iii) of the Theorem is proved at the end of $\S 3$.

Existence of generalized $\mathcal{K}$-sets (defined below), $K_{p}$-sets, and Kronecker sets is established in $\S 2$. The properties of tensor algebras needed are summarized in $\S 3$. The corollaries are proved in $\S 4$.

Let $G$ be a locally compact abelian group, and let $\Gamma$ denote the dual group of $G$. $A(G)$ is the Fourier algebra of $G$. For a compact subset $E$ of $G, A(E)$ denotes the set of restrictions of elements of $A(G)$ to $E$, each restriction being given the quotient norm.

Corollary 1.2. Let $G$ be a compact abelian group and let $E \subset G$ be compact. If $E$ contains the sum of two perfect compact subsets, then $A(E)$ is not Arens regular.

A Banach algebra $A$ is "Arens regular" [1] if the two Arens multiplications on $A^{* *}$ agree. The preceding corollary slightly strengthens a result implicit in [10. p. 213] by replacing "is the sum of disjoint perfect subsets of a $\mathcal{K}$-set" with "contains the sum of two perfect sets". Granirer [5, Cor. 6] proves a stronger form of the following corollary.

Corollary 1.3. If $E \subset \mathbb{R}$ (or $E \subset \mathbb{T}$ ) is symmetric, then $A(E)$ is not Arens regular.

A symmetric set is a set of the form $E=\left\{\sum_{j=1}^{\infty} \epsilon_{j} x_{j}: \epsilon_{j}=0,1\right\} \subset \mathbb{R}$, where $x_{k}>0$ and $x_{k}>\sum_{j>k} x_{j}$ for all $k \geq 1$. (Granirer's conclusions are stronger, but with some effort we could obtain more from the fact that $A(E)$ has a quotient that contains $A\left(D_{2}\right)$.)

Corollary 1.4. Let $G$ be a locally compact abelian group and let $E \subset G$ be compact. If $E$ contains the sum of two perfect compact subsets, then $M(E)$ is not norm dense in $A(E)^{*}$.

$M(E)$ is the set of regular Borel measures concentrated on $E$. This contrasts with the situation when $E=F_{1}+F_{2}$ is the sum of two countable compact sets. Then $M(E)$ is norm dense in $P M(E)=A(E)^{*}$.

Definition 1.5. A Kronecker set [12 p. 97] (or $K_{o}$-set [6, p. 552]) is a closed $E \subset G$ such that every continuous function from $E$ to the unit circle is the uniform limit, on $E$, of continuous characters.

A $K_{p}$-set [6, p. 552], [12, p. 98] is a closed $E \subset G$ such that every continuous function from $E$ to the $p$-th roots of unity is a continuous character.

A $\mathcal{K}$-set is a set that is either a Kronecker set or a $K_{p}$-set for some $p$. $\mathbb{Z}$

A subset $E \subset G$ is independent if whenever $n \geq 1, x_{1}, \ldots, x_{n} \in E, m_{1}, \ldots, m_{n} \in$

$$
\sum_{j} m_{j} x_{j}=0 \Rightarrow m_{1} x_{1}=\cdots=m_{n} x_{n}=0 .
$$

A Kronecker set is independent; so is a $K_{p}$-set if all of its elements have order $p$ (see Lemma 2.7). The converse is spectacularly false; see, e.g., [9]. 
A Cantor set is a non-empty, perfect, and totally disconnected (subset of a) metrizable space. Every Cantor set is homeomorphic to the Cantor middle-third set. Every relatively open subset of a Cantor set $X$ contains a Cantor set 1

For $2 \leq q<\infty, D_{q}$ will denote the countably infinite product of the $q$-th roots of unity with themselves, with the product topology. $D_{2}$ is often called the "Walsh" group. Each $D_{q}$ is perfect, totally disconnected, and metrizable, hence a Cantor set.

We let $\mathbb{T}_{p}$ be the $p$-th roots of unity.

The dual group of the general locally compact abelian group $G$ will be denoted by $\Gamma$.

Finally, we need one new term for this paper. A generalized $\mathcal{K}$-set $X$ in a locally compact abelian group is a compact set $X$ which is a finite, disjoint union $X=X_{1} \cup \cdots \cup X_{n}$ such that each $X_{j}$ is either a Kronecker or $K_{p}$-set (the $p$ 's can vary) and such that the following condition holds (compare 12, 5.1.3]). For each $\epsilon>0$ and each continuous function $f: X \rightarrow \mathbb{T}$ with the property that $f\left(X_{j}\right) \subset T_{p_{j}}$ if $X_{j}$ is a $K_{p_{j}}$-set, there exists a continuous character $\gamma$ on $G$ such that $|\langle\gamma, x\rangle-f(x)|<\epsilon$ on $X$.

The only novelty in the next proposition is "generalized $\mathcal{K}$-set". Its proof is a variation on standard arguments. See [13, 4.2.1-4.2.2], 6, 42.10] or [4, 11.1.2] for those.

Proposition 1.6. Let $X=\bigcup_{j=1}^{n} X_{j}$ be a generalized $\mathcal{K}$-set. Then

$$
A\left(X_{1}+\cdots+X_{n}\right) \cong C\left(X_{1}\right) \hat{\otimes} \cdots \hat{\otimes} C\left(X_{n}\right) \text {. }
$$

2. Finding KRoneCKer And $K_{p}$-SETS AND THE PROOF OF PARTS (i)-(ii) OF THE THEOREM

Let $X_{1}^{\prime}, \ldots, X_{n}^{\prime}$ be Cantor subsets of the metrizable locally compact abelian group $G$ and let $d(\cdot, \cdot)$ be the metric on $G$. Let $E_{1}, \ldots, E_{n}$ be disjoint Cantor subsets of $\mathbb{T}$. Let $U$ be the set of continuous functions from $E=\bigcup_{j=1}^{n} E_{j} \rightarrow X^{\prime}=$ $\bigcup_{j=1}^{n} X_{j}^{\prime}$ that map $E_{j}$ into $X_{j}^{\prime}$ for $1 \leq j \leq n$. Then $U$ is a complete metric space under the uniform norm, $D(f, g)=\sup _{x \in E} d(f(x), g(x))$. The following proposition generalizes [7, pp. 91-92] and [13, 4.3.3]. Taking an $f$ for which $Y_{j}=f\left(E_{j}\right)$ is perfect for all $j$, the proposition proves parts (i)-(ii) of the Theorem stated in the Abstract.

Proposition 2.1. Let $X_{1}, \ldots, X_{n}$ be Cantor subsets of the metrizable locally compact abelian group $G$. Then there exist Cantor subsets $X_{j}^{\prime} \subset X_{j}$, and $g_{1}, \ldots, g_{n}$, $1 \leq j \leq n$, such that for quasi-all $f \in U$ such that $f\left(E_{j}\right) \subset X_{j}^{\prime}$ for all $j$, the following hold:

(1) $\bigcup_{j=1}^{n}\left(f\left(E_{j}\right)-g_{j}\right)$ is a generalized $\mathcal{K}$-set;

(2) for each $1 \leq j \leq n$, either $f\left(E_{j}\right)$ is a perfect Kronecker set or there is some $2 \leq p_{j}$ such that $f\left(E_{j}\right)-g_{j}$ is a perfect $K_{p_{j}}$-set all of whose elements have order $p_{j}$; and

(3) for those $j$ 's for which $K_{j}$ is a Kronecker set, $g_{j}=0$.

\footnotetext{
${ }^{1}$ We can see this by noting that $X$, being a Cantor set, is homeomorphic to the Cantor middlethird set. Of course, every subset of the middle-third set with non-empty interior contains a Cantor subset. Hence, so does every subset of $X$ that has interior.
} 
The Proposition is false (see Example 2.2 (iii) below) without the restriction to the $X_{j}^{\prime}$ 's.

We prove Proposition 2.1 at the end of this section.

The following examples indicate why the $g_{j}$ are needed and why we need some of the complications that follow.

Example 2.2. (i) There are sets that are translates of $K_{p}$-sets (for some $p>2$ ), but which contain no $K_{p}$-set (for any $p$ ) nor any Kronecker set. Here is one. Let $G=\mathbb{T}^{\infty}$, the countably infinite product of $\mathbb{T}$ with itself. Then $G$ contains a copy of $D_{p}$ for all $2 \leq p<\infty$. Furthermore, $G$ contains an element $g$ such that $\{n g: n \in \mathbb{Z}\}$ is dense in $G$ (this follows easily from [12, 2.33]: $G$ is a quotient of the Bohr compactification of $\mathbb{Z}$ ). Let $X$ be a perfect $K_{2}$-set inside $D_{2} \subset G$. Let $Y=X+g$. We claim that $Y$ is not a $K_{p}$-set, whatever the $p$. Let $\gamma$ be a continuous character on $G$. Then $\langle\gamma, x+g\rangle= \pm\langle\gamma, g\rangle$, for $x \in X$. Since $g$ generates $G,\langle\gamma, g\rangle$ cannot be a root of unity, whatever the non-zero $\gamma \in \Gamma$. Therefore, for each $2 \leq p<\infty$, there is no continuous character $\gamma$ on $G$ such that $\langle\gamma, Y\rangle=\left\{e^{2 \pi i / p}\right\}$. Hence, $Y$ is not a $K_{p}$-set. Since the difference of any two elements of $Y$ has order two, $Y$ cannot contain a Kronecker set.

(ii) [6, 41.16] Let $x \in \mathbb{T}$ have infinite order, and let $X$ be a perfect $K_{2}$-set in $D_{2}$. Consider $Y=\{x\} \times X \subset \mathbb{T} \times D_{2}$. Then $Y$ is a $K_{2}$-set, none of whose elements have order 2. Hence, $Y$ is not independent. Also, $Y$ has no $K_{p}$-subsets for $p>2$ and no Kronecker subsets, aside from singletons.

The translate of a $K_{2}$-set $X$ in $D_{4}$ by an element $g$ of order 4 with $2 g \notin X$ will yield another $K_{p}$-set $Y=X+g$ whose elements have (the artificial) order 4 , and so $Y$ is not quite independent.

(iii) Let $X_{1}$ be a perfect Kronecker set in $\mathbb{T}$, let $x \neq y \in X_{1}$, and let $X_{2}$ be a perfect $K_{2}$-set in $D_{2}$. Consider

$$
X=\left(X_{1} \times\{0\}\right) \cup\left(\{x\} \times X_{2}\right) \cup\left(\{y\} \times X_{2}\right) \subset \mathbb{T} \times D_{2} .
$$

Then $X$ is a disjoint union of Cantor sets, but $X$ is not a generalized $\mathcal{K}$-set. Indeed, let $a \neq b$ be any two complex numbers of absolute value one, neither of which is in $\{ \pm 1\}$. Then continuous characters do not approximate the "local character" that is $a$ on $X_{1} \times\{0\}, a$ on $\{x\} \times X_{2}$ and $b$ on $\{y\} \times X_{2}$, even though the "unionees" of $X$ are a Kronecker set and two $K_{2}$-sets.

Definition 2.3. For $2 \leq \ell<\infty$, let $G_{\ell}$ denote the closure of the group generated by the set of elements of $G$ of order exactly $\ell$.

Let $X \subset G$ and let $k(X)$ be the smallest integer $\ell$ such that a coset $y+G_{\ell}$ contains a perfect compact subset of $X$. If there is no such $\ell$, we let $k(X)=\infty$. That $k(X)$ is well defined is evident.

The definition of $k(X)$ can be understood as a generalization of the $\left(m_{1}, m_{2}\right)$ notion of 13 .

The next three lemmas give useful properties of the mapping $X \mapsto k(X)$.

Lemma 2.4. Let $X$ be a Cantor subset of the metrizable locally compact abelian group $G$ and let $v \in G$. Let an integer $m \geq 2$ be given, and let $F: X \rightarrow G$ be given by $x \mapsto m x+v$. Suppose that for some $z \in G, Y=F^{-1}(z)$ contains a Cantor set (e.g., if $Y$ has non-empty relative interior in $X)$. Then $k(X) \leq m$. 
Proof of Lemma 2.4. Let $X^{\prime}$ be a Cantor subset of $X$ such that $F\left(X^{\prime}\right)=\{z\}$. Then for all $x \neq x^{\prime} \in X^{\prime}$,

$$
\begin{aligned}
0 & =z-z \\
& =m x+v-\left(m x^{\prime}+v\right) \\
& =m\left(x-x^{\prime}\right) .
\end{aligned}
$$

Fix $x^{\prime} \in X^{\prime}$ and let $X^{\prime \prime}$ be a Cantor subset of $X^{\prime}$ that does not contain $x^{\prime}$. Then

$$
m\left(X^{\prime \prime}-x^{\prime}\right)=0 .
$$

That is, $X^{\prime \prime}-x^{\prime} \subset G_{m}$ and so $X^{\prime \prime} \subset G_{m}+x^{\prime \prime}$. Hence, $k(X) \leq m$.

Lemma 2.5. Let $X$ be a Cantor subset of the metrizable locally compact abelian group $G$. If $k(X)=\infty$, then $X$ contains a Cantor subset, all of whose elements have infinite order.

Proof of Lemma 2.5. A Baire category argument implies that $\bigcup_{k} X \cap G_{k}$ is first category in $X$. Therefore a standard Cantor set construction will find a Cantor subset $X^{\prime}$ with $X^{\prime} \cap G_{k}=\emptyset$ for all $k \geq 2$.

For the proof of Proposition 2.1 we will need the following lemma, which is a slight strengthening of [6, 41.3-4], replacing "open" with "Cantor". The proof essentially follows [6, 41.3-4], uses Lemma 2.4 and Lemma 2.5, and is omitted.

Lemma 2.6. Let $G$ be a metrizable compact abelian group. Let $X$ be a Cantor subset of $G$. Let $W_{1}, \ldots, W_{n}$ be Cantor subsets of $X$ (e.g., closed-open subsets of $X)$.

Suppose that, for each $1 \leq j \leq n$, either

(1) $k\left(W_{j}\right)=\infty$ or

(2) that there exist $2 \leq p_{j}<\infty$ with $k\left(W_{j}\right)=p_{j}$ and $W_{j} \subset G_{p_{j}}$.

Then there exist $x_{1} \in W_{1}, \ldots, x_{n} \in W_{n}$ such that $\left\{x_{1}, \ldots, x_{n}\right\}$ is independent.

The next lemma is well known [12, 5.1.3]; combined with Lemma 2.6, items (1)(2) say that finite Kronecker and $K_{p}$-sets exist in profusion, and item (3) says that each finite independent set is a generalized $\mathcal{K}$-set.

Lemma 2.7. Let $X$ be a finite independent subset of the locally compact abelian group $G$. Then

(1) If the elements of $X$ all have infinite order, $X$ is a Kronecker set.

(2) If $2 \leq p<\infty$ and the elements of $X$ all have order $p$, then $X$ is a $K_{p}$-set.

(3) If $f: X \rightarrow \mathbb{T}$ is such that for all $x$, Order $(x)=p<\infty$ implies $f(x) \in \mathbb{T}_{p}$, then for all $\epsilon>0$, there exists $\gamma \in \Gamma$ with

$$
|\langle x, \gamma\rangle-f(x)|<\epsilon
$$

for all $x \in X$.

Proof of Proposition 2.1. If the $X_{j}$ are not pairwise disjoint, we replace them by smaller perfect subsets that are pairwise disjoint. (This is easy.) Then, for each $1 \leq j \leq n$, we do the following:

(1) We let $p_{j}=k\left(X_{j}\right)$.

(2) If $p_{j}=\infty$, we let $X_{j}^{\prime}$ be a Cantor subset of $X_{j}$ such that all elements of $X_{j}^{\prime}$ have infinite order. This can be done by Lemma 2.5. We let $g_{j}=0$. 
(3) If $p_{j}<\infty$, we find $g_{j} \in G$ and a Cantor set $X_{j}^{\prime} \subset X_{j}$ such that $X_{j}^{\prime}-g_{j} \subset$ $G_{p_{j}}$.

(4) Let $Y_{j}=X_{j}^{\prime}-g_{j}$.

Then, after reordering the $Y_{j}$ if necessary, we are in the following situation:

(5) all $Y_{j}, 1 \leq j \leq n$, are Kronecker sets; or

(6) for each integer $j \in[1, n]$ there exists $2 \leq p_{j}<\infty$ such that $Y_{j}$ is a $K_{p_{j}}$-set and all elements of $Y_{j}$ have order $p_{j}$; or

(7) there is some $1 \leq m<n$ such that $Y_{1}, \ldots, Y_{m}$ are Kronecker sets, and for $m<j \leq n$ there exists $2 \leq p_{j}<\infty$ such that $Y_{j}$ is a $K_{p_{j}}$-set all of whose elements have order $p_{j}$.

Let $U_{o}$ be the set of continuous functions from $E=\bigcup_{j=1}^{n} E_{j} \rightarrow Y=\bigcup_{j=1}^{n} Y_{j}$ that map $E_{j}$ into $Y_{j}$ for $1 \leq j \leq n$. Then it will suffice to prove that quasi-all $f \in U_{o}$ are such that

(8) $f\left(E_{j}\right)$ is perfect for $1 \leq j \leq n$;

(9) $f(E)$ is a generalized $\mathcal{K}$-set, and, more precisely,

(10) for all $\gamma_{1}, \ldots, \gamma_{n} \in \Gamma$, and $\epsilon>0$, there exists $\gamma \in \Gamma$ with

$$
\left|\langle\gamma, z\rangle-\left\langle\gamma_{j}, z\right\rangle\right|<\epsilon \text { for } z \in f\left(E_{j}\right) \text { and } 1 \leq j \leq n .
$$

We give the proof, assuming (7). We leave the reader to give the separate proofs under the assumptions of (5), and under (6).

We first prove (8). We show that quasi-all $f \in U_{o}$ have perfect range on $E$. Let $\left\{W_{\ell}\right\}$ be a countable neighbourhood basis for $E$ with all $W_{\ell}$ closed and open. Consider the set

$$
S(\ell)=\left\{f \in U_{o}: f\left(W_{\ell}\right) \text { is a singleton }\right\} .
$$

It is easy to see that $S(\ell)$ has no interior. Also, $S(\ell)$ is closed. Indeed, suppose that $f_{k} \rightarrow f$ uniformly on $E$. Let $f_{k}\left(W_{\ell}\right)=\left\{x_{k}\right\}$. Then there is a subsequence such that $x_{k(j)} \rightarrow x$, since $Y$ is compact. A two- $\epsilon$ argument shows that $f\left(W_{\ell}\right)=\{x\}$. That is enough to show $S(\ell)$ is closed. Then $\bigcup_{\ell} S(\ell)$ is first category, and so quasi-all $f \in U_{o}$ have perfect ranges on $E$. This proves (8).

The proof of (9)-(10) follows [7 pp. 90-92] very, very closely.

The metric on $G$ is $d(\cdot, \cdot)$, as noted. Because $Y=\bigcup_{1}^{n} Y_{j}$ and $E=\bigcup_{1}^{n} E_{j}$ are Cantor sets, the locally constant functions $f: E \rightarrow Y$ are uniformly dense in $U$. Let $\phi: Y \rightarrow \mathbb{T}$ be locally constant, and with

$$
\phi(z) \in \mathbb{T}_{p_{j}} \text { when } z \in E_{j} \text { and } m<j \leq n .
$$

Let $\epsilon>0$. Let $A(\phi, \epsilon)$ be the set of $f \in U_{o}$ such that

$$
\gamma \in \Gamma \Rightarrow \sup _{x \in E}|\langle\gamma, f(x)\rangle-\phi(f(x))| \geq \epsilon .
$$

Then $A(\phi, \epsilon)$ is a closed subset of $U_{o}$. We claim that $A(\phi, \epsilon)$ has no interior in $U_{o}$. We argue by contradiction. Suppose that $W$ is an open subset contained in $A(\phi, \epsilon)$. Then there exist $f \in W$ which is locally constant on $E$ and $\rho>0$ such that $g \in U_{o}$ and

$$
\sup _{x \in E} d(f(x), g(x))<\rho \Rightarrow g \in W .
$$

We may assume that $\rho$ is smaller than the minimum of the distances between the sets $X_{j}, 1 \leq j \leq n$, and smaller than the minimum of the distances between the elements of $f(E)$. 
By Lemma 2.6, we can find a finite independent set

$$
\left\{x_{\ell}: 1 \leq \ell \leq r\right\} \subset Y
$$

that is $\rho / 4$-dense in $Y$.

Let $f(E)=\left\{y_{1}, \ldots, y_{k}\right\}$. For each $1 \leq s \leq k$, let $\ell(s)$ be any integer in $[1, \ldots, r]$ such that $d\left(x_{\ell(s)}, y_{s}\right)<\rho / 4$. Such $\ell(s)$ exists by the $\rho / 4$-density of the $x_{\ell}$. The $\rho / 4$ density also implies that each $x_{\ell(s)}$ belongs to the same $Y_{j}$ as $y_{s}$. Hence, $\operatorname{Order}\left(x_{\ell(s)}\right)=\operatorname{Order}\left(y_{s}\right)$.

We can choose $h \in U_{o}$ such that

$$
f(z)=y_{s} \Rightarrow h(z)=x_{\ell(s)} .
$$

Because $f$ is locally constant, so is $h$. Furthermore, $\sup _{x \in E} d(f(x), h(x)) \leq \rho / 4$. Since the image of $h$ is independent, Lemma 2.7 (3) implies that (2.3) fails. Hence, $h \notin A(\phi, \epsilon)$, and $A(\phi, \epsilon)$ is nowhere dense.

Now let $\phi_{\ell}$ be a sequence of locally constant functions from $X$ to $\mathbb{T}$ that is dense in the space of continuous functions from $Y \rightarrow \mathbb{T}$ that satisfy (2.2). Then

$$
R=\bigcup_{\ell=1, m=1}^{\infty} A\left(\phi_{\ell}, 1 / m\right)
$$

is first category in $U_{o}$. Thus, if $f \in U_{o}$ and $f \notin R$, then for every locally constant, continuous $\phi: f(E) \rightarrow \mathbb{T}$ satisfying $\phi(x) \in \mathbb{T}_{p_{j}}$ if $\operatorname{Order}(x)=p_{j}$ (that is, (2.2)), we have

$$
\inf _{\gamma \in \Gamma} \sup _{x \in f(E)}|\langle\gamma, x\rangle-\phi(x)|=0 .
$$

It now follows that (2.1) holds, and that $f(E)$ is a perfect generalized $\mathcal{K}$-set, with $f\left(E_{j}\right)$ a perfect Kronecker set if $j \leq m$ and a perfect $K_{p_{j}}$-set if $m<j$. This proves (9)-(10).

\section{Tensor algebra Background}

AND THE PROOF OF PART (iii) OF THE THEOREM

Let $X_{1}, \ldots, X_{n}$ be locally compact Hausdorff spaces. The projective tensor product of the $C_{o}\left(X_{j}\right)$ is the space

$$
V=V\left(X_{1}, \ldots, X_{n}\right)=C_{o}\left(X_{1}\right) \hat{\otimes} \ldots \hat{\otimes} C_{o}\left(X_{n}\right)
$$

consisting of all sums

$$
f=\sum_{j=k}^{\infty} h_{1, j} \otimes \cdots \otimes k_{n, j}, k_{n, j} \in C_{o}\left(X_{j}\right),
$$

subject to

$$
\sum_{j=1}^{\infty}\left(\prod_{\ell=1}^{n}\left\|h_{\ell, j}\right\|_{\infty}\right)<\infty .
$$

The norm $\|f\|=\|f\|_{V}$ is the infimum of the quantities (3.3) subject to (3.2).

Now consider the special case $n=2$ and $X_{1}=X_{2}=D_{2}$. We will use the locally compact abelian group structure of $D_{2}$. Let $M: C\left(D_{2}\right) \rightarrow C\left(D_{2} \times D_{2}\right)$ be given by $M f(x, y)=f(x+y)$. Then it is easy to show that $M$ maps $A\left(D_{2}\right)$ into $V\left(D_{2}, D_{2}\right)$, and $M$ is isometric and multiplicative.

We have the following conclusions: 
$V\left(D_{2}, D_{2}\right)$ is not Arens regular, since Arens regularity is inherited by subalgebras and quotient algebras, and $A\left(D_{2}\right)$ is not Arens regular [2].

Of course, since $V\left(D_{2}, \ldots, D_{2}\right)$ ( $n \geq 3$ terms) has $V\left(D_{2}, D_{2}\right)$ as a quotient, $V\left(D_{2}, \ldots, D_{2}\right)$ is not Arens regular.

For further tensor algebra background, see, e.g., 4, Chapter 11] for more related results, further bibliography, applications, and history.

Proof of part (iii) of the Theorem. By Proposition 2.1, we can find elements $g_{1}$, $\ldots, g_{n} \in G$ and a generalized $\mathcal{K}$-set $X^{\prime}=\bigcup\left(X_{j}^{\prime}-g_{j}\right)$ with $X_{j}^{\prime}$ a Cantor subset of $X_{j}$ for $j=1, \ldots, n$. By Proposition 1.6, $A\left(X_{1}^{\prime}+\cdots+X_{n}\right) \cong V\left(X_{1}^{\prime}, \ldots, X_{n}^{\prime}\right)$. Since $A(F) \cong A(F+g)$ for all closed $F \subset G$ and $g \in G, A\left(X_{1}^{\prime}+g_{1}+\cdots+X_{n}^{\prime}+g_{n}\right) \cong$ $A\left(X_{1}^{\prime}+\cdots+X_{n}\right)$.

\section{Proofs of the corollaries}

Proof of Corollary 1.2 when $G$ is metrizable. $A(E)$ has the quotient

$$
A\left(E_{1}+F_{1}\right) \cong C\left(E_{1}\right) \hat{\otimes} C\left(F_{1}\right) \cong C\left(D_{2}\right) \hat{\otimes} C\left(D_{2}\right)=V\left(D_{2}, D_{2}\right) .
$$

Since regularity is inherited by sub- and quotient algebras, and $V\left(D_{2}, D_{2}\right)$ is not Arens regular, $A(E)$ is not Arens regular.

Proof of Corollary 1.2, general case. We reduce to the case of metrizable $G$. Suppose $F_{1}+F_{2} \subseteq E$, where $F_{1}, F_{2}$ are compact and perfect. Let $A_{1}=\left\{x_{1,2}, x_{1,2}\right\} \subset F_{1}$ and $B_{1}=\left\{y_{1,1}, y_{1,2}\right\} \subset F_{2}$. Choose a finite set of characters $\Lambda_{1} \subset \Gamma$ such that the elements of $\Lambda_{1}$ separate the points of $A_{1} \cup B_{1}$. Let $H_{1}$ denote the annihilator of the closed group generated by $\Lambda_{1}$. If the images in $G / H_{1}$ of both $F_{1}$ and $F_{2}$ contain perfect sets, we cease the construction and replace $G$ with $G / H_{1}$ and $E, F_{1}, F_{2}$ with their canonical images in $G / H_{1}$. Otherwise, we have begun the induction.

Assume that $\ell \geq 1$ and that for $1 \leq k \leq \ell$,

(i) $A_{k}=\left\{x_{k, 1}, \ldots, x_{k, 2^{k}}\right\} \subset F_{1}$ and $B_{k}=\left\{y_{k, 1}, \ldots, y_{k, 2^{k}}\right\} \subset F_{2}$ have been chosen so that (if $k>1$ ) $A_{k-1} \subset A_{k}, B_{k-1} \subset B_{k}$;

(ii) $\Lambda_{k-1} \subset \Lambda_{k} \subset \Gamma$ are such that $\Lambda_{k}$ separates $A_{k} \cup B_{k}$;

(iii) $H_{\ell}$ is the annihilator of the closed group generated by $\Lambda_{\ell}$; and

(iv) for $1<k \leq \ell$, for each $x \in A_{k}$ (resp. $x \in B_{k}$ ) there is a different $x^{\prime} \in A_{k}$ (resp. $y^{\prime} \in B_{k}$ ) such that for all $\lambda \in \Lambda_{k-1}$,

$$
\begin{aligned}
& \left|\langle\lambda, x\rangle-\left\langle\lambda, x^{\prime}\right\rangle\right|<2^{-k}, \text { resp. } \\
& \left|\langle\lambda, y\rangle-\left\langle\lambda, y^{\prime}\right\rangle\right|<2^{-k} .
\end{aligned}
$$

If the images in $G / H_{\ell}$ of both $F_{1}$ and $F_{2}$ contain perfect sets, we cease the construction and replace $G$ with $G / H_{\ell}$ and $E, F_{1}, F_{2}$ with their canonical images in $G / H_{\ell}$.

We let $\Lambda=\bigcup_{j=1}^{\infty} \Lambda_{j}$ and $H$ be the annihilator of $\Lambda$. Then by (4.1), the images $X$ (resp. $Y$ ) of $F_{1}$ (resp. $F_{2}$ ) in $G / H$ contain perfect sets. Of course, $G / H$ is metrizable.

Since Arens regularity would be passed from $A(E)$ to $A(X+Y)$ (the latter is a subalgebra of a quotient algebra of the former), it is enough to prove that $A(X+Y)$ is not Arens regular; that is, we may assume that $G$ is metrizable.

We now apply the proof for metrizable $G$. 
Proof of Corollary 1.3. It is easy to see that a symmetric set $E$ is the sum of the two sets

$$
\begin{aligned}
& E_{1}=\left\{\sum_{j=1}^{\infty} \epsilon_{2 j} x_{2 j}: \epsilon_{j}=0,1\right\} \text { and } \\
& E_{2}=\left\{\sum_{j=1}^{\infty} \epsilon_{2 j+1} j x_{2 j+1}: \epsilon_{j}=0,1\right\} .
\end{aligned}
$$

Now apply Corollary 1.2 .

Proof of Corollary 1.4. If $M(E)$ were dense in $A(E)^{*}$, then $A(E)$ is Arens regular, by [3, 4.1]. Since $A(E)$ is not Arens regular, by Corollary 1.2, Corollary 1.4 follows.

\section{REFERENCES}

1. R. Arens, The adjoint of a bilinear operation, Proc. Amer. Math. Soc. 2 (1951), 839-848. MR 13:659f

2. P. Civin and B. Yood, The second conjugate space of a Banach algebra as an algebra, Pacific J. Math 11 (1961), 847-870. MR 26:622

3. C. C. Graham, Arens regularity and the second dual of certain quotients of the Fourier algebra, Quart. J. Math. 52 (2001), 13-24. MR 2002d:43002

4. C. C. Graham and O. C. McGehee, Essays in Commutative Harmonic Analysis, SpringerVerlag, New York, 1979. MR 81d:43001

5. E. E. Granirer, Day points for quotients of the Fourier algebra $A(G)$, extreme nonergodicity of their duals and extreme non-Arens regularity, Illinois J. Math. 40 (No. 3) (1996), 402-419. MR 98c: 43005

6. E. Hewitt and K. A. Ross, Abstract Harmonic Analysis I, II, Springer-Verlag, Berlin, Göttingen, Heidelberg, 1963, 1970. MR 28:158, MR 41:7378

7. J. P. Kahane, Séries de Fourier Absolument Convergentes, Springer-Verlag, Berlin, Göttingen, Heidelberg, 1970. MR 43:801

8. R. Kaufman, A functional method for linear sets, Israel J. Math. 5 (1967), 185-187. MR 38:4902

9. T. W. Körner, A pseudofunction on a Helson set, I and II, Astérisque 5 (1973), 2-224, and 231-239. MR 53:8772a. MR 53:8772c

10. F. Lust-Piquard, Élements ergodiques et totalement ergodiques dans $L^{\infty}(G)$, Studia Math. 69 (1981), 191-225. MR 84h:43003

11. Y. Meyer, Recent Advances in Spectral Synthesis, Conference on Harmonic Analysis, Maryland, 1971, Lecture Notes in Mathematics 266, Springer-Verlag, Berlin, 1972, pp. 239-253. MR 52:14863

12. W. Rudin, Fourier Analysis on Groups, Wiley Interscience, New York, 1962. MR 27:2808

13. N. Th. Varopoulos, Tensor algebras and harmonic analysis, Acta Math. 119 (1968), 51-112. MR 39:1911

Department of Mathematics, University of British Columbia, RR\#1-H-46, Bowen Island, British Columbia, CAnada V0N 1G0

E-mail address: ccgraham@alum.mit.edu 